Article

\title{
Enhanced Light Extraction Efficiency and Modulation Bandwidth of Deep-Ultraviolet Light-Emitting Diodes with Al Nanospheres
}

\author{
Xiaolong Hu ${ }^{1}$, Xu Liang ${ }^{1}$, Lingyun Tang ${ }^{1}$ and Wenjie Liu ${ }^{2, *}$ \\ 1 School of Physics and Optoelectronics, South China University of Technology, Guangzhou 510640, China; \\ scxlhu@scut.edu.cn (X.H.); phxl@mail.scut.edu.cn (X.L.); sctangly@scut.edu.cn (L.T.) \\ 2 School of Information Engineering, Guangdong University of Technology, Guangzhou 510006, China \\ * Correspondence: wjliu@gdut.edu.cn
}

check for

updates

Citation: Hu, X.; Liang, X.; Tang, L.; Liu, W. Enhanced Light Extraction Efficiency and Modulation Bandwidth of Deep-Ultraviolet Light-Emitting Diodes with $\mathrm{Al}$ Nanospheres. Crystals 2022, 12, 289. https://doi.org/10.3390/cryst 12020289

Academic Editors: Degang Zhao and Baoping Zhang

Received: 30 December 2021 Accepted: 16 February 2022 Published: 18 February 2022

Publisher's Note: MDPI stays neutral with regard to jurisdictional claims in published maps and institutional affiliations.

Copyright: (c) 2022 by the authors. Licensee MDPI, Basel, Switzerland. This article is an open access article distributed under the terms and conditions of the Creative Commons Attribution (CC BY) license (https:/ / creativecommons.org/licenses/by/ $4.0 /)$.

\begin{abstract}
Planar, nanopillar and Al nanosphere structure AlGaN-based deep-ultraviolet lightemitting diodes (DUV-LEDs) were numerically investigated via a three-dimensional finite difference time domain (3D FDTD) method. The three types of DUV-LEDs were compared and analyzed in terms of light extraction efficiency (LEE), Purcell factor $\left(F_{P}\right)$ and modulation bandwidth. The results showed that nanopillar structure DUV-LEDs with optimal nanopillar height, width and spacing can enhance transverse electric (TE)-polarized LEE to 39.7\% and transverse magnetic (TM)-polarized LEE to $4.4 \%$. The remarkable improvement was mainly due to the increased scattering effect, decreased absorption of the p-GaN layer and total internal reflection (TIR) effect. After adopting the $\mathrm{Al}$ nanospheres, the TE-polarized modulation bandwidth was increased by $71 \mathrm{MHz}$ and the TMpolarized LEE was enhanced approximately 4.3-fold as compared to the nanopillar LED structure, while the $\mathrm{Al}$ nanosphere diameter was $120 \mathrm{~nm}$. The reasons for promotion are mainly attributed to the coupling behavior of diploe and localized surface plasmon induced by $\mathrm{Al}$ nanospheres. The designed structures provide a meaningful solution for realization of high-efficiency DUV-LEDs.
\end{abstract}

Keywords: DUV-LEDs; localized surfaced plasmon; light extraction efficiency; modulation bandwidth

\section{Introduction}

In recent years, AlGaN-based deep-ultraviolet (DUV) light-emitting diodes (LEDs) have been greatly developed because of their wide-ranging applications, including water and air purification [1], disinfection [2] and DUV communications [3]. Nevertheless, the performance of traditional planar DUV-LEDs is limited due to low external quantum efficiency (EQE) for shorter emission wavelengths [4,5]. EQE is the product of two independent factors: the internal quantum efficiency (IQE) and the light extraction efficiency (LEE). The LEE of DUV-LEDs is only in the range of 7-9\% [6], and contributes to poor EQE. There are many factors that affect the LEE. On the one hand, there is strong light absorption in the $\mathrm{p}-\mathrm{GaN}$ layer of DUV-LEDs. On the other hand, as the $\mathrm{Al}$ component in AlGaN-based quantum gradually increases [7], transverse magnetic (TM) polarization of DUV-LEDs accounts for the main part of transverse electric (TE) polarization. In addition, it is difficult for the photons to escape from the device due to the strong total internal reflection (TIR) effect. Therefore, improving LEE of DUV-LEDs is necessary, and several strategies have been proposed including nanowire structure [8], patterned sapphire substrates [9], surface roughing [10] and photonic crystal patterns [11]. Another effective way that placing metal nanoparticles on the $\mathrm{p}-\mathrm{GaN}$ contact layer to stimulate localized surface plasmons (LSPs) coupling behavior is beneficial is that it enhances spontaneous emission rate and light extraction [12-14]. Based on the above methods, LEE can be effectively extracted. In addition, it is reported by Lee et al. in 2020 that the internal quantum efficiency is enhanced by $57.7 \%$ due to the LSPs for the $\mathrm{Al}$ nanospheres adjacent to the active region [15]. Furthermore, the 
DUV-LEDs are also used in light communications $[16,17]$. High modulation bandwidth is important for UV communication with high data transmission rate. It is recently reported that the modulation bandwidth of DUV-LEDs is $153 \mathrm{MHz}$ [16]. At present, there are reports on the relationship among spontaneous emission, modulation speed, and surface plasmons only for the InGaN-based LEDs $[18,19]$. In fact, apart from the LEE, $F_{P}$ related to spontaneous emission and modulation bandwidth of the device are extremely important for AlGaN-based DUV-LEDs. However, simultaneous discussion for LEE, Purcell factor $\left(F_{P}\right)$, and modulation bandwidth of InGaN-based LEDs has been reported [20], but has not been investigated for AlGaN-based LEDs. How to enhance these optical characteristics for DUV-LEDs is also worthy of study.

In this work, nanopillar and Al nanosphere structure DUV-LEDs were designed and numerically investigated by FDTD simulation method. In terms of nanopillar structure DUV LEDs, the optimized parameters include the nanopillar height, width and spacing. The purpose was to improve the LEE of LED by decreasing the absorption of p-GaN layer, TIR effect and enhancing the scattering effect. For the Al nanosphere structure, QW-LSP coupling effect induced by $\mathrm{Al}$ nanospheres have intention of improving the overall LEE, $F_{P}$ and LEE of TE and TM-polarized DUV-LEDs. Moreover, the modulation bandwidths of planar and proposed structures are theoretically calculated and demonstrated. The optical characteristics, such as LEE, $F_{P}$ and modulation bandwidth, are carefully discussed for nanopillar and Al nanosphere structure DUV-LEDs.

\section{Device Structures and Parameters}

The simulated structures of the DUV-LEDs were carried by using a three-dimensional finite difference time-domain (3D FDTD) method, which solves the differential forms of Maxwell's equations to calculate electromagnetic field distributions [21]. Figure 1 shows a schematic diagram for three different DUV-LEDs. In Figure 1a, a simulated model of the planar DUV-LEDs is displayed. The layer structures were composed of a sapphire substrate, a 1000-nm-thick n-AlGaN layer, 50-nm-thick AlGaN multiple quantum wells (MQWs) active layers, a 100-nm-thick p-AlGaN layer, and a 10-nm-thick GaN layer. Meanwhile, if there were five quantum wells, the thickness of the total quantum well was $50 \mathrm{~nm}$, and a single dipole source was at the center of the quantum well, which is $25 \mathrm{~nm}$. The spectrum of the dipole source was a Gaussian shape, and the center wavelength and full width at half maximum of the spectrum were $270 \mathrm{~nm}$ and $40 \mathrm{~nm}$, respectively. For the polarized dipole source, the dipole in the $x$ - and $z$-axis directions represent TE polarization and TM polarization, respectively. The refractive index of materials for the sapphire layer, the AlGaN layer and GaN layer was set at 1.8, 2.6 and 2.9, respectively, [22,23], the absorption coefficients of the GaN layer, the AlGaN layer and MQWs were assumed to be $170,000 \mathrm{~cm}^{-1}, 10 \mathrm{~cm}^{-1}$ and $1000 \mathrm{~cm}^{-1}$. The large absorption coefficient caused light to be strongly absorbed in the GaN layer. The dimension of the simulated structure was $1000 \mathrm{~nm} \times 1000 \mathrm{~nm} \times 800 \mathrm{~nm}$ for $\mathrm{x}-, \mathrm{y}$ - and $\mathrm{z}$-directions, respectively. The boundary conditions of the simulation area were set as a perfectly matched layer (PML), which absorbed electromagnetic energy. The mesh of the simulation model was set as $5 \mathrm{~nm}$ to ensure its accuracy to calculate the quantum efficiency. A power box monitor was placed near the dipole source to obtain the dipole-radiated power, and an $x-y$ plane power monitor at $100 \mathrm{~nm}$ above the $\mathrm{p}-\mathrm{GaN}$ layer was placed to obtain the radiation power of light escaping from the upper layer to the air interface. The value of LEE was defined as the ratio of the total extracted power collected from the power monitor to the dipole-radiated power. The Purcell factor $\left(F_{P}\right)$ was defined as the ratio of the corresponding powers from the dipole inside the device and the emitter inside bulk material [24]. $F_{P}$ was obtained from the dipole source via the FDTD method. Figure $1 \mathrm{~b}$ describes the nanopillar structure of the DUV-LEDs. As compared to the planar structure, there were three other parameters change: the height $(\mathrm{H})$, the width $(\mathrm{w})$ of the nanopillar and the spacing $(\mathrm{d})$ between adjacent nanopillars, which had an important impact on LEE and $F_{P}$ for the nanopillar structure of DUV-LEDs. A similar structure with different material layers has also been reported in 
InGaN-based LEDs [25]. Metal nanoparticles were placed into the nanopillar structure as shown in Figure 1c, and Al nanospheres were used for enhancing LEE due to effectively scattering [26], reducing p-GaN absorption, improving TM-polarized emission, and could be used for inducing an LSP coupling effect at a deep ultraviolet spectral range [27]. Thus, an $\mathrm{Al}$ nanosphere structure was selected, and their diameters were equal to the spacing between two adjacent nanopillars (d). Since metal $\mathrm{Al}$ is easily oxidized, the $\mathrm{Al}$ nanosphere structure was composed of a $3 \mathrm{~nm}$ thick $\mathrm{Al}_{2} \mathrm{O}_{3}$ shell with $\mathrm{Al}$ as the core. Therefore, $\mathrm{LEE}, F_{P}$ and the modulation bandwidth of the three DUV-LEDs structures will be analyzed with the aid of the FDTD method.

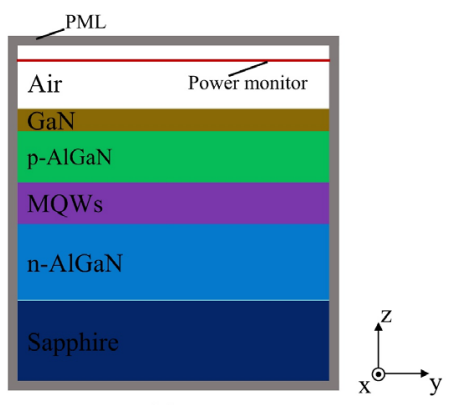

(a)

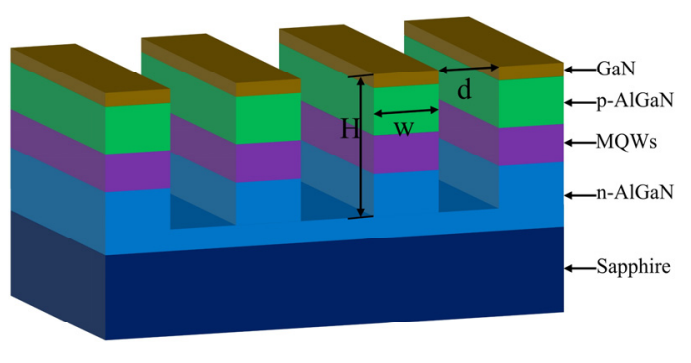

(b)

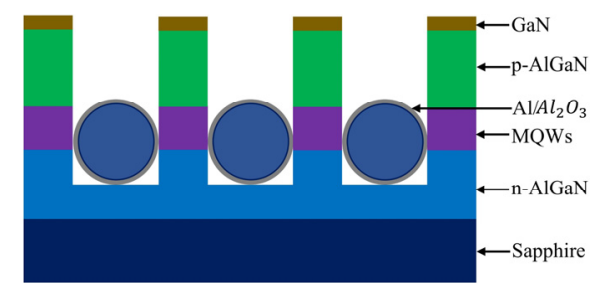

(c)

Figure 1. Schematic diagrams of the simulated (a) planar structure, (b) nanopillar structure and (c) $\mathrm{Al}$ nanosphere structure.

\section{Results and Discussion}

\subsection{Planar Structure}

This section depicts simulation results of planar structure DUV-LEDs. As shown in Figure 2, LEE and $F_{P}$ for the planar structure DUV-LEDs in TE-polarized and TM-polarized light were calculated with various wavelengths ranging from 250 to $290 \mathrm{~nm}$. Note that the dashed and solid lines in the picture represent TE and TM polarization, and the black and red curves indicate LEE and $F_{P}$ of the DUV-LED, respectively. Within the emission wavelength change, the curves are flat and there is almost no fluctuation for LEE in different polarization. However, in terms of TE-polarized LEE, it is low and approximately $4.2 \%$, mainly due to the absorption of the p-GaN layer and the TIR effect. At the same time TM-polarized LEE is even lower at below $0.5 \%$, the reason being that the total TIR effect becomes much stronger, and it is more difficult for the TM-polarized photons. LEE for TM polarization is about one tenth that for TE polarization, and the TM-polarized emission dominates in the AlGaN MQWs especially for the deep ultraviolet wavelength. In addition, the two red curves indicate that when the emission wavelength increases, TE-polarized $F_{P}$ and TM-polarized $F_{P}$ both increase from approximately 1.1 to 1.23 . It is found that the LEE and $F_{P}$ of the planar structure DUV-LEDs are low. Therefore, improvement of LEE and $F_{P}$ become very urgent. 


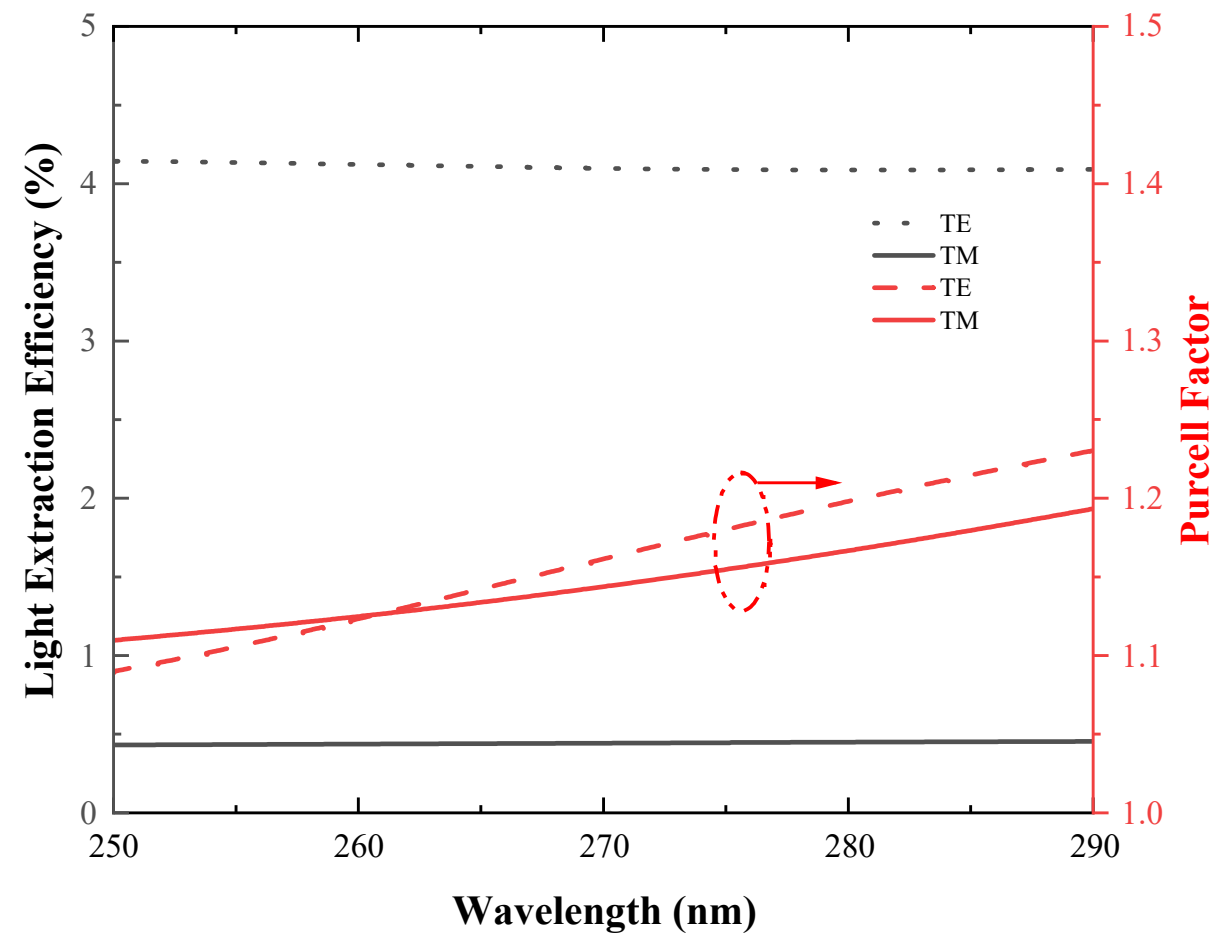

Figure 2. TE- and TM-polarized light extraction efficiency and Purcell factor within the wavelength ranging from $250 \mathrm{~nm}$ to $290 \mathrm{~nm}$.

\subsection{Nanopillar Structure}

In order to enhance the TE-polarized and TM-polarized LEE, the DUV-LEDs nanopillar structures are explored. The schematic diagram of the nanopillar structure DUV-LEDs is shown in Figure 1b. Three key parameters of nanopillar structure include nanopillar height, width and spacing between adjacent nanopillars, which are optimized to increase LEE. Figure 3 shows TE-polarized and TM-polarized LEE of DUV-LEDs nanopillar structure with various nanopillar height. The selected emission wavelength is $270 \mathrm{~nm}$ and the nanopillar height is modified from $110 \mathrm{~nm}$ to $240 \mathrm{~nm}$. The nanopillar widths of $20 \mathrm{~nm}$, $30 \mathrm{~nm}$ and $40 \mathrm{~nm}$ were chosen to match the emission wavelength. Moreover, the period of nanopillar was set to $100 \mathrm{~nm}, 120 \mathrm{~nm}$ and $140 \mathrm{~nm}$, which represent the sum of the nanopillar width and the spacing of adjacent nanopillar. The epilayers including the $\mathrm{p}-\mathrm{GaN}$ layer, the p-AlGaN layer, the MQWs layer and part of n-AlGaN layer were designed as nanopillar shapes. Figure 3 a depicts that TE-polarized LEE with various nanopillar height and width. Note that the LEE is approximately $6 \%$ when the nanopillar height is less than $140 \mathrm{~nm}$. It is observed that the LEE was enhanced significantly as the height increases in the range of 140-210 $\mathrm{nm}$ for all DUV-LEDs. Then the LEE began to decrease while the height of nanopillar continued to increase. TE-polarized LEE reached the maximum at $39.4 \%$ when the nanopillar height was $210 \mathrm{~nm}$, the width was $20 \mathrm{~nm}$ and the spacing was $100 \mathrm{~nm}$. Compared with planar DUV-LEDs, there is a great improvement, about nine-fold, for TE-polarized LEE in terms of nanopillar structure. Similarly, a. TM-polarized LEE with the same parameter changes is shown in Figure $3 \mathrm{~b}$. As the nanopillar height increased, the TM-polarized LEE starts increased, then slowly decreased, and finally remained stable. However, when the nanopillar width was $20 \mathrm{~nm}$, TM-polarized LEE kept improving when the nanopillar height exceeded $200 \mathrm{~nm}$. Furthermore, the highest TM-polarized LEE was $7.2 \%$ when the nanopillar height, width and spacing were $240 \mathrm{~nm}, 20 \mathrm{~nm}$ and $120 \mathrm{~nm}$, respectively. TM-polarized LEE of the nanopillar structure was 18 times higher than that of the planar DUV-LEDs. In addition, the TE-polarized LEE reached its maximum which the nanopillar height, width and spacing were $210 \mathrm{~nm}, 20 \mathrm{~nm}$ and $100 \mathrm{~nm}$, respectively. At the same time, the TM-polarized LEE also reached $4.4 \%$ and greatly improved compared to 
planar DUV-LEDs, which means more photons could escape and be collected. On the one hand, the filling area of the $\mathrm{p}-\mathrm{GaN}$ layer was reduced, and as a result of the absorption was reduced; on the other hand, there was more possibility for the light generated by the dipole source to escape to the air interface, breaking the TIR effect and increasing the scattering effect.
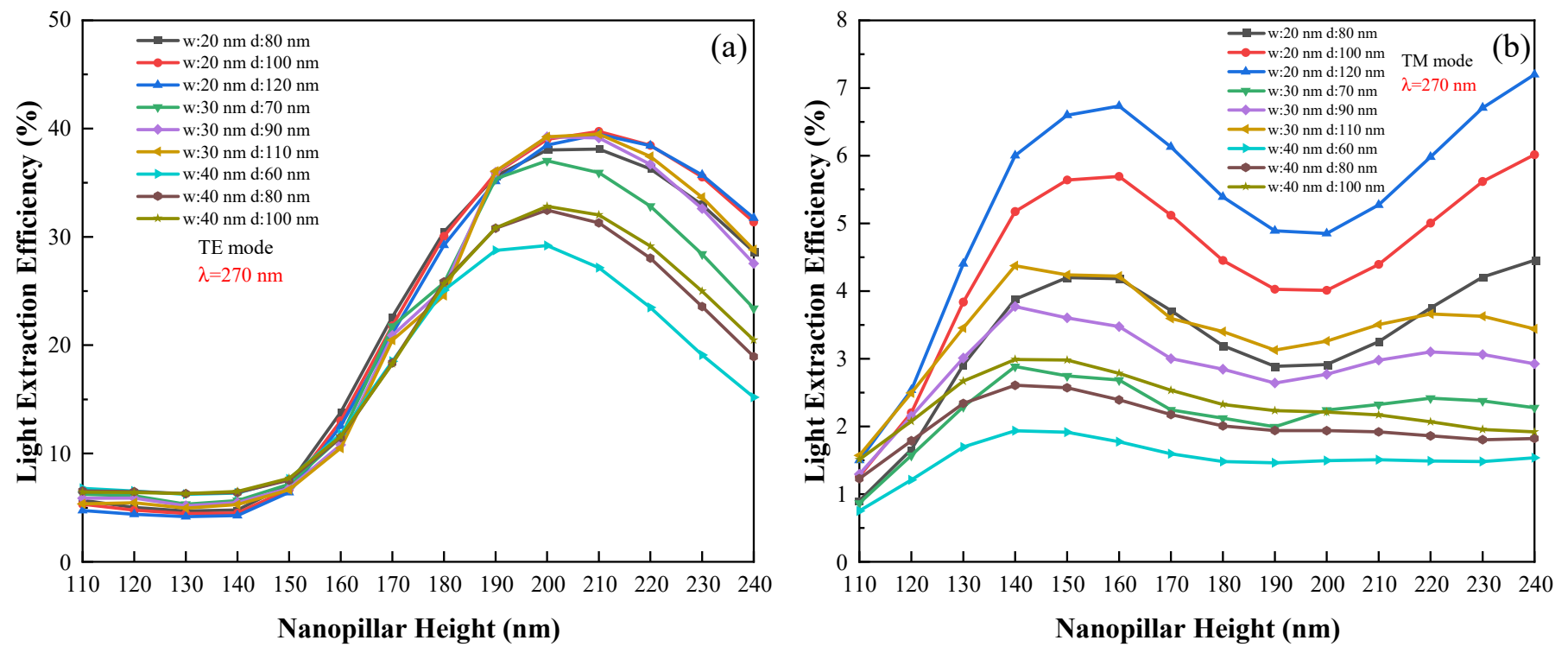

Figure 3. LEE as a function of nanopillar height and widths were set to 20, 30 and $40 \mathrm{~nm}$ for (a) TE-polarization and (b) TM-polarization with emission wavelength of $270 \mathrm{~nm}$.

TE-polarized far-field patterns for planar DUV-LEDs and the nanopillar structure with a height of $210 \mathrm{~nm}$ are shown in Figure $4 \mathrm{a}, \mathrm{b}$, respectively. It can be observed that the electric intensity of the DUV-LEDs with nanopillar structure is stronger and is an order of magnitude higher than that of the planar DUV-LEDs. The strong electric field intensity distribution means that more photons could escape and be extracted, leading to an improvement in LEE. It is speculated that the proposed nanopillar method would improve the scattering effect. In addition, the TE and TM-polarized $F_{P}$ of the nanopillar structure DUV-LEDs are investigated, and the results are shown in Table 1.

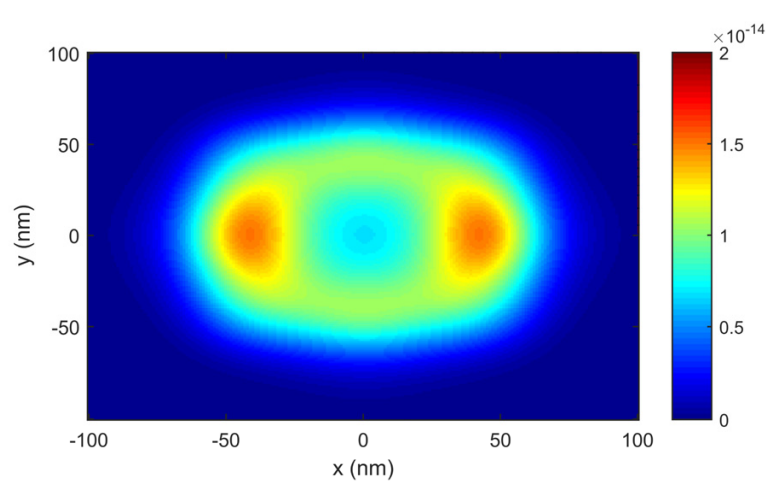

(a)

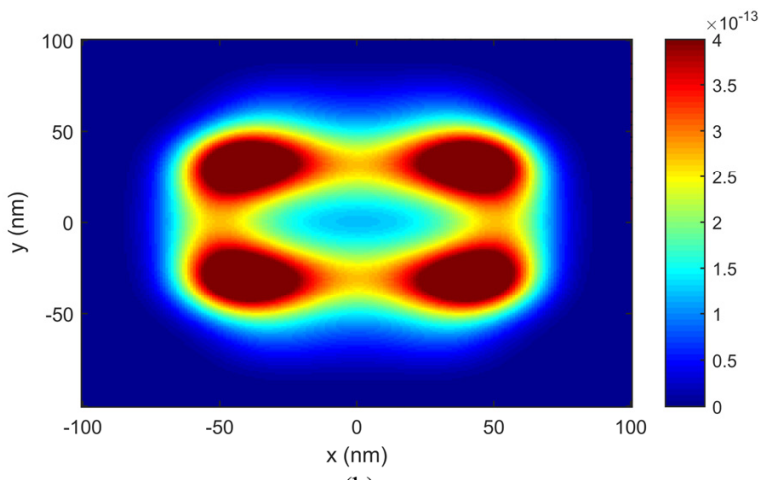

(b)

Figure 4. Far-field radiation patterns at (a) planar DUV-LEDs and (b) nanopillar structure with height of $170 \mathrm{~nm}$ and width of $20 \mathrm{~nm}$. 
Table 1. Comparison on different polarized LEE, Purcell factor and modulation bandwidth for planar, nanopillar and Al nanosphere structure DUV-LEDs.

\begin{tabular}{|c|c|c|c|c|}
\hline Structure & Polarization & LEE (\%) & $F_{P}$ & Bandwidth (MHz) \\
\hline \multirow{2}{*}{ Planar } & $\mathrm{TE}$ & 4.1 & 1.162 & 375.30 \\
\hline & $\mathrm{TM}$ & 0.4 & 1.144 & 374.97 \\
\hline \multirow{2}{*}{ Nanopillar } & $\mathrm{TE}(\mathrm{H}=210 \mathrm{~nm})$ & 39.7 & 0.186 & 357.13 \\
\hline & $\mathrm{TM}(\mathrm{H}=210 \mathrm{~nm})$ & 4.4 & 0.924 & 370.88 \\
\hline \multirow{4}{*}{ Al nanosphere } & $\mathrm{TE}(\mathrm{H}=180 \mathrm{~nm} \mathrm{D}=120 \mathrm{~nm})$ & 20.7 & 4.009 & 428.30 \\
\hline & $\mathrm{TM}(\mathrm{H}=180 \mathrm{~nm} \mathrm{D}=120 \mathrm{~nm})$ & 2.1 & 1.666 & 384.69 \\
\hline & $\mathrm{TE}(\mathrm{H}=240 \mathrm{~nm} \mathrm{D}=120 \mathrm{~nm})$ & 9.4 & 0.348 & 360.16 \\
\hline & $\mathrm{TM}(\mathrm{H}=240 \mathrm{~nm} \mathrm{D}=120 \mathrm{~nm})$ & 19.1 & 1.123 & 374.58 \\
\hline
\end{tabular}

\subsection{Al Nanosphere Structure}

Here, $\mathrm{Al}$ nanospheres were placed into the nanopillar structure, as shown in Figure 1c. Photons and excitons in MQWs were effectively scattered to the air interface by $\mathrm{Al}$ nanospheres with the right coupling condition. Thus, it is beneficial to the improvement of LEE and $F_{P}$ for DUV-LEDs. In particular, the diameter of the Al nanosphere was equal to the spacing between the adjacent nanopillar. The purpose of this was to be more conductive to coupling with $\mathrm{Al}$ nanosphere and MQWs when the coupling distance was shortened. Figure $5 \mathrm{a}, \mathrm{b}$ show TE-polarized LEE and $F_{P}$ for the $\mathrm{Al}$ nanosphere structure with various diameters, from $80 \mathrm{~nm}$ to $140 \mathrm{~nm}$, when the nanopillar height was set to $180 \mathrm{~nm}, 200 \mathrm{~nm}$, $220 \mathrm{~nm}$ and $240 \mathrm{~nm}$, respectively. When the nanopillar height was $180 \mathrm{~nm}$ or $240 \mathrm{~nm}$, it had a better enhancement in the TE-polarized LEE. However, the results for high $F_{P}$ were at the nanopillar heights of $180 \mathrm{~nm}$ or $200 \mathrm{~nm}$. Therefore, a reasonable height is $180 \mathrm{~nm}$ for high TE-polarized LEE and $F_{P}$. When the $\mathrm{Al}$ nanosphere diameter was $110 \mathrm{~nm}$, the LEE reached $24.1 \%$ and $F_{P}$ was 3.7. TE-polarized LEE and $F_{P}$ were increased approximately 6 times and 3 times simultaneously as compared to the planar DUV-LED. At different nanopillar heights, LEE and $F_{P}$ varied greatly with diameter of $\mathrm{Al}$ nanospheres, mainly due to various spacing between the dipole source and $\mathrm{Al}$ nanospheres. This resulted in different localized surface plasmon intensities. When the nanopillar height was $180 \mathrm{~nm}$, the LSP effect was significant, and improvement of LEE and $F_{P}$ was also obvious. Furthermore, the $F_{P}$ of $\mathrm{Al}$ nanosphere structure was enlarged by more than 20 times compared to the nanopillar structure for TE-polarized DUV-LEDs. Both LEE and $F_{P}$ of TE polarization were significantly improved.
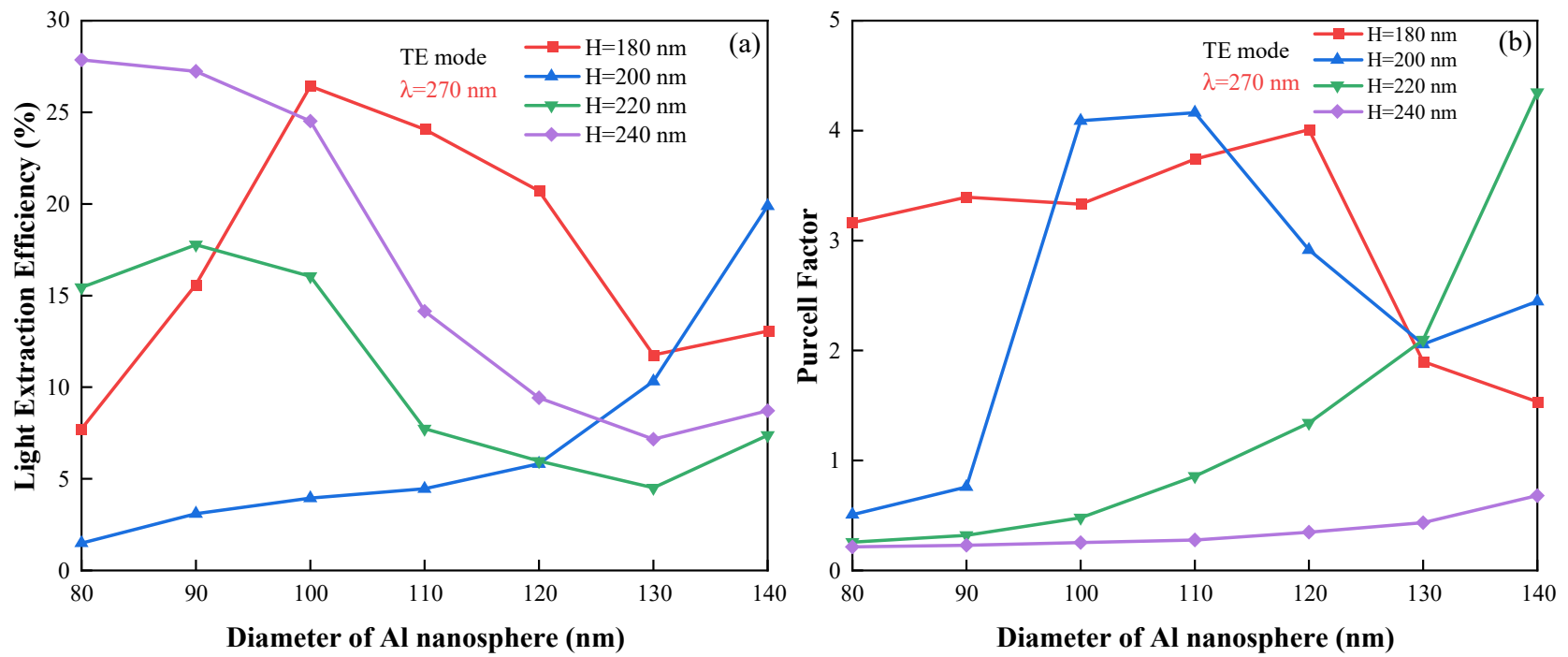

Figure 5. TE-polarized (a) LEE and (b) Purcell factor of the structure with various nanopillar heights as a function of diameter of $\mathrm{Al}$ nanosphere. 
Similarly, the TM-polarized LEE and $F_{P}$ of Al nanosphere structure are exhibited in Figure $6 a, b$, respectively. TM-polarized LEE significantly increased under Al nanosphere structure, when the height was $200 \mathrm{~nm}, 220 \mathrm{~nm}$ and $240 \mathrm{~nm}$. Moreover, when the Al nanosphere diameter was $120 \mathrm{~nm}$ and the height was $240 \mathrm{~nm}$, the maximum LEE of TM polarization was $19.1 \%$. Compared with nanopillar structure, LEE was enhanced four-fold, and the corresponding $F_{P}$ was not reduced. Except for the diameter of $130 \mathrm{~nm}, F_{P}$ had a large increase, and it remained stable under other $\mathrm{Al}$ nanosphere diameters. In the $\mathrm{Al}$ nanosphere structure, the LEE of TM polarization was extremely improved. Furthermore, it is inferred that coupling $\mathrm{Al}$ nanospheres and MQWs with LSP mode can generate obvious improvement of LEE, because nanopillar structure increased the scattering effect and broke the limit of TIR. For TM-polarized LEE and $F_{P}$, with different nanopillar heights, the LSP coupling strength of $\mathrm{Al}$ nanospheres and the dipole source was different. It led to a high LEE when the nanopillar height was $240 \mathrm{~nm}$. $F_{P}$ had a tendency to increase due to the volume reduction when nanopillar height increased.
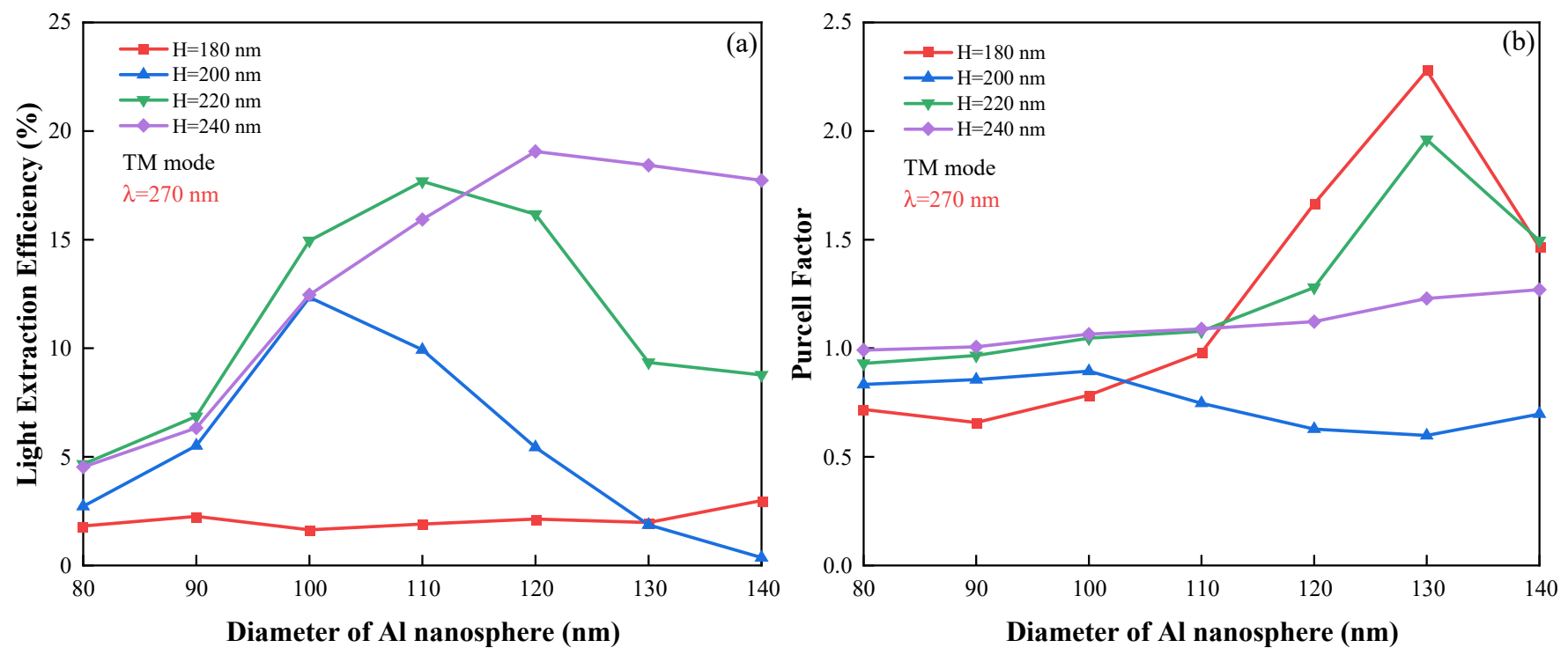

Figure 6. TM-polarized (a) LEE and (b) Purcell factor of the structure with various nanopillar heights as a function of diameter of $\mathrm{Al}$ nanosphere.

Figure 7 shows the electric field intensity distribution for the nanopillar and $\mathrm{Al}$ nanosphere structure DUV-LEDs in TM polarization with 240-nm-high nanopillar height and 120-nm-wide nanosphere diameter. A notable increase of local electromagnetic field along the edge of $\mathrm{Al}$ nanospheres can be seen, and the strongest enhancement occurs at the interface between $\mathrm{Al}$ nanospheres and the nanopillar. The electric field with $\mathrm{Al}$ nanospheres distributes more widely, and more light escapes into the air, increasing the LEE compared to the nanopillar structure. This confirms that the increase in TM-polarized LEE comes from strong QW-LSP coupling process. 


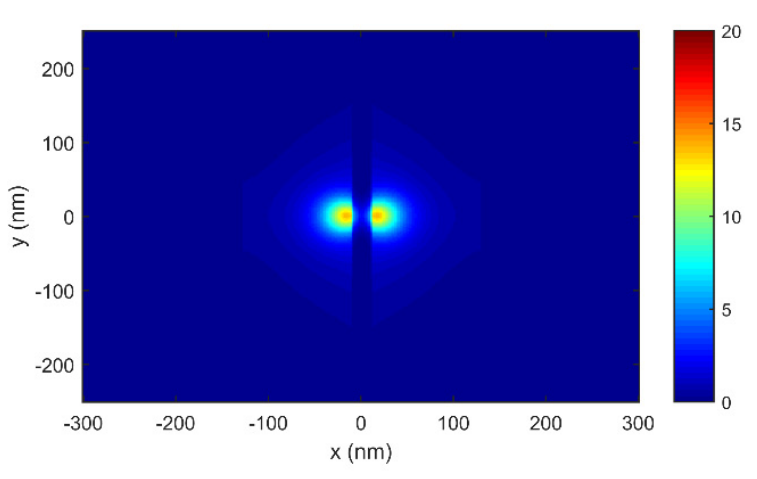

(a)

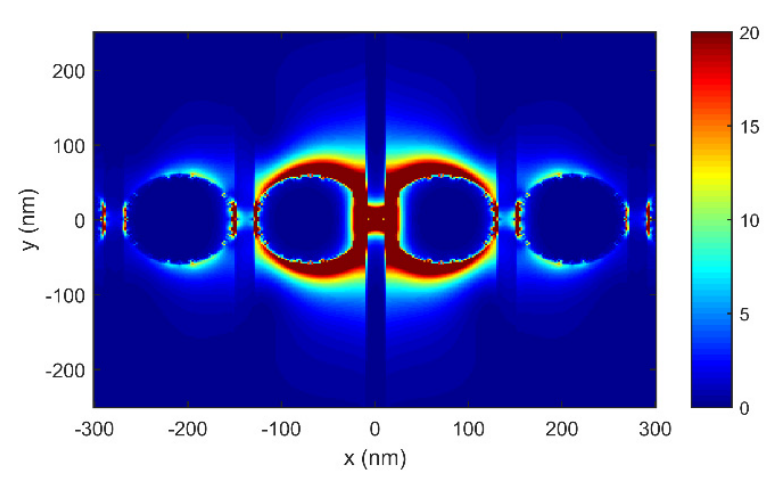

(b)

Figure 7. Electric field distributions for (a) nanopillar and (b) Al nanosphere structure DUV-LEDs with nanopillar height being $230 \mathrm{~nm}$.

\subsection{Modulation Bandwidth}

Finally, in order to study the influence of nanopillar and QW-LSP coupling on the spontaneous emission rate, modulation bandwidth of DUV-LEDs is analyzed. In terms of carriers in QW LEDs, the effective carrier lifetime modified by Purcell effect can be expressed as [28]:

$$
\frac{1}{\tau_{e f f}}=\frac{F_{P}}{\tau_{r}}+\frac{1}{\tau_{n r}}
$$

where $\tau_{\text {eff }}$ is the effective recombination lifetime, $\tau_{r}$ is radiative recombination lifetime, $\tau_{n r}$ is the nonradiative recombination lifetime, for a bi-molecular recombination mechanism, from [29] the modulation bandwidth is expressed as:

$$
f_{3 \mathrm{~dB}}=\frac{1}{2 \pi \tau_{\text {eff }}}
$$

The radiative recombination lifetime of planar LED is selected as $\tau_{r}=5.8 \mathrm{~ns}$, which is measured in [30]. Using light induced transient gratins (LITG) transients' technology, the non-radiative recombination lifetime is obtained for $\tau_{n r}=0.45 \mathrm{~ns}$ as studied in [31]. According to the above Formulas (1) and (2), the radiation recombination lifetime parameters and simulated $F_{P}$ value, we evaluated the modulation bandwidth for the designed DUV-LEDs. Figure 8 shows the modulation bandwidth of Al nanosphere structure with various diameter, as the nanopillar height is $180 \mathrm{~nm}$ and $240 \mathrm{~nm}$. The LEE is relatively high at this height. Note that both TE and TM polarization achieve high modulation bandwidth at the height of $180 \mathrm{~nm}$. The modulation bandwidth reaches $428.3 \mathrm{MHz}$ for TE polarization at the diameter of $120 \mathrm{~nm}$ and $396 \mathrm{MHz}$ for TM polarization at the diameter of $130 \mathrm{~nm}$. It is observed that DUV-LEDs with design of Al nanosphere structure can achieve high LEE, $F_{P}$ and significantly enhance modulation bandwidth.

In addition to the above calculation of the modulation bandwidth of Al nanosphere structure, Table 1 presents a comparison of TE-polarized and TM-polarized LEE, $F_{P}$ and modulation bandwidths of three types of DUV-LEDs. It is found that the LEE of the planar structure is very low. Under the optimization of the nanopillar structure, the LEE of TE polarization was greatly improved but hindered by poor $F_{P}$. Moreover, the proposed $\mathrm{Al}$ nanosphere structure can simultaneously improve the LEE, $F_{P}$ and modulation bandwidth. For DUV-LEDs with Al nanospheres, the TE-polarized modulation speed increased by 71 $\mathrm{MHz}$ compared to the nanopillar structure. The modulation speed remained stable for TM polarization, but the LEE was greatly enhanced and reached 19\% due to the QW-LSP coupling effect. More details show that the addition of Al nanospheres not only improves LEE of DUV-LED but also increases its modulation speed. The designed nanopillar and Al nanosphere structure are helpful to improve the overall performance of DUV-LEDs. 


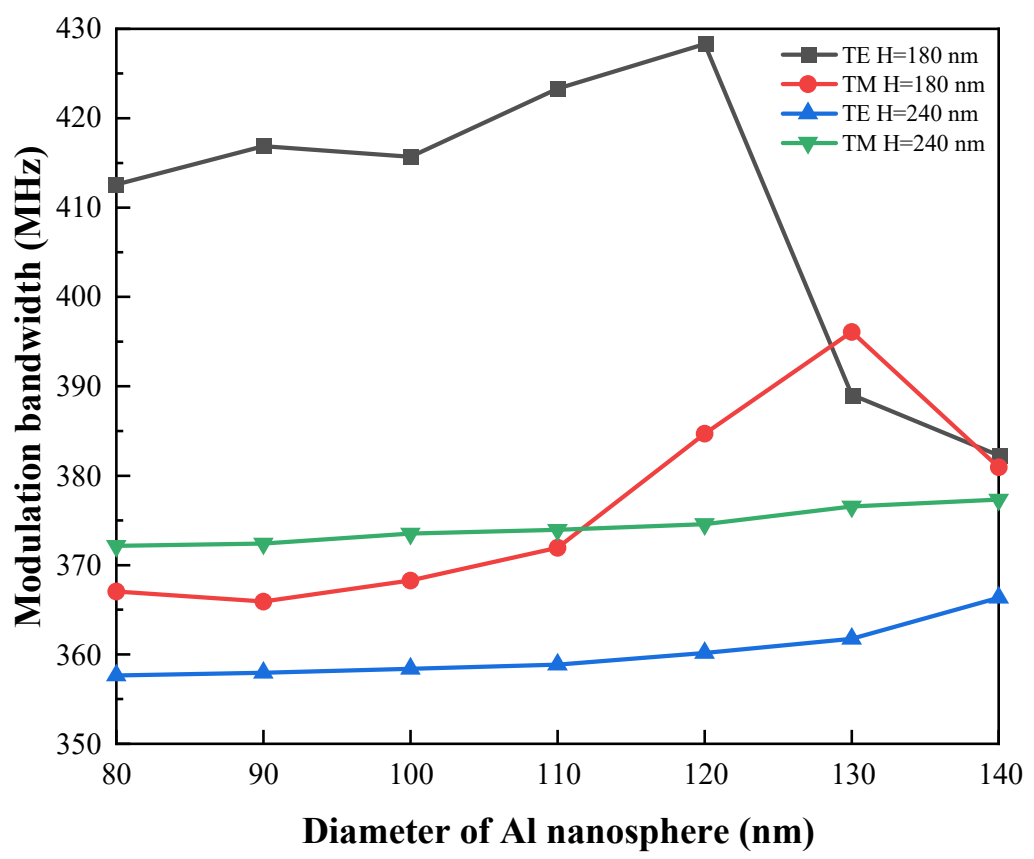

Figure 8. TE and TM polarized modulation bandwidth of Al nanosphere structure DUV-LEDs with various nanopillar heights as a function of diameter of $\mathrm{Al}$ nanosphere.

\section{Conclusions}

In summary, TE and TM- polarized LEE, $F_{P}$ and modulation bandwidths of planar, nanopillar and Al nanosphere structure DUV-LEDs, respectively, were simulated using the FDTD method. Relative to the planar structure DUV-LED, the designed nanopillar structure with various heights, width and spacing greatly improved the TE and TM-polarized LEE. The three key parameters played important roles in enhancing LEE by contributing to reducing the absorption of the $\mathrm{p}-\mathrm{GaN}$ layer, raising the scattering ability and interrupting the TIR effect. The results indicate that TE-polarized LEE is 39.7\% and TM-polarized LEE is $4.4 \%$. An $\mathrm{Al}$ nanosphere structure is proposed on the basis of nanopillar structure. It is proved that LEE, $F_{P}$ and modulation bandwidths of both polarizations are enhanced at the same time by QW-LSP coupling induced on $\mathrm{Al}$ nanospheres. When $\mathrm{Al}$ nanospheres' diameter was $120 \mathrm{~nm}$, TE-polarized modulation bandwidth speed reached $428.3 \mathrm{MHz}, \mathrm{TM}-$ polarized LEE was $19.1 \%$. Compared with the planar structure DUV-LEDs, TM-polarized LEE was improved 48-fold for $\mathrm{Al}$ nanosphere structure DUV-LEDs. It is believed that the $\mathrm{Al}$ nanosphere structure is a superior solution for achieving high-efficiency and highbandwidth DUV-LEDs in the future.

Author Contributions: Conceptualization, X.H. and X.L.; methodology, X.H. and X.L.; validation, X.H.; formal analysis, X.L. and L.T.; investigation, X.L.; resources, W.L.; writing, X.H. and X.L.; visualization, X.L. and L.T.; supervision, X.H. and W.L. All authors have read and agreed to the published version of the manuscript.

Funding: This research was funded by the National Natural Science Foundation of China (61975037); Natural Science Foundation of Guangdong Province (2019A1515010905 and 2019A1515011471).

Institutional Review Board Statement: Not applicable.

Informed Consent Statement: Not applicable.

Data Availability Statement: All supporting data and computational details are available on written request. These data are stored by the main author of this article.

Conflicts of Interest: The authors declare no conflict of interest. 


\section{References}

1. Shur, M.S.; Gaska, R. Deep-Ultraviolet Light-Emitting Diodes. IEEE Trans. Electron Devices 2010, 57, 12-25. [CrossRef]

2. Wurtele, M.A.; Kolbe, T.; Lipsz, M.; Kulberg, A.; Weyers, M.; Kneissl, M.; Jekel, M. Application of GaN-based ultraviolet-C light emitting diodes-UV LEDs-for water disinfection. Water Res. 2011, 45, 1481-1489. [CrossRef] [PubMed]

3. He, X.; Xie, E.; Islim, M.S.; Purwita, A.A.; McKendry, J.J.D.; Gu, E.; Haas, H.; Dawson, M.D. 1 Gbps free-space deep-ultraviolet communications based on III-nitride micro-LEDs emitting at $262 \mathrm{~nm}$. Photonics Res. 2019, 7, B41. [CrossRef]

4. Kneissl, M.; Seong, T.-Y.; Han, J.; Amano, H. The emergence and prospects of deep-ultraviolet light-emitting diode technologies. Nat. Photonics 2019, 13, 233-244. [CrossRef]

5. Hirayama, H.; Maeda, N.; Fujikawa, S.; Toyoda, S.; Kamata, N. Recent progress and future prospects of AlGaN-based high-efficiency deep-ultraviolet light-emitting diodes. Jpn. J. Appl. Phys. 2014, 53, 100209. [CrossRef]

6. Kneissl, M.; Kolbe, T.; Chua, C.; Kueller, V.; Lobo, N.; Stellmach, J.; Knauer, A.; Rodriguez, H.; Einfeldt, S.; Yang, Z.; et al. Advances in group III-nitride based deep UV light-emitting diode technology. Semicond. Sci. Technol. 2010, 26, 014036. [CrossRef]

7. Kawanishi, H.; Niikura, E.; Yamamoto, M.; Takeda, S. Experimental energy difference between heavy- or light-hole valence band and crystal-field split-off-hole valence band in AlxGa1-xN. Appl. Phys. Lett. 2006, 89, 251107. [CrossRef]

8. Djavid, M.; Mi, Z. Enhancing the light extraction efficiency of AlGaN deep ultraviolet light emitting diodes by using nanowire structures. Appl. Phys. Lett. 2016, 108, 051102. [CrossRef]

9. Dong, P.; Yan, J.; Zhang, Y.; Wang, J.; Geng, C.; Zheng, H.; Wei, X.; Yan, Q.; Li, J. Optical properties of nanopillar AlGaN/GaN MQWs for ultraviolet light-emitting diodes. Opt. Express 2014, 22 (Suppl. 2), A320-A327. [CrossRef]

10. Lee, C.; Cheng, B.; Lee, Y.; Kuo, H.; Lu, T.; Wang, S. Output power enhancement of vertical-injection ultraviolet light emitting diodes by GaN-free and surface roughness structures. Electrochem. Solid-State Lett. 2009, 12, H44. [CrossRef]

11. Oder, T.N.; Kim, K.H.; Lin, J.Y.; Jiang, H.X. III-nitride blue and ultraviolet photonic crystal light emitting diodes. Appl. Phys. Lett. 2004, 84, 466-468. [CrossRef]

12. Kuo, Y.; Chen, H.T.; Chang, W.Y.; Chen, H.S.; Yang, C.C.; Kiang, Y.W. Enhancements of the emission and light extraction of a radiating dipole coupled with localized surface plasmon induced on a surface metal nanoparticle in a light-emitting device. Opt. Express 2014, 22 (Suppl. 1), A155-A166. [CrossRef] [PubMed]

13. Wang, J.; Yang, G.; Zhang, Q.; Gao, S.; Zhang, R.; Zheng, Y. Localized Surface Plasmon-Enhanced Deep-UV Light-Emitting Diodes with $\mathrm{Al} / \mathrm{Al}_{2} \mathrm{O}_{3}$ Asymmetrical Nanoparticles. Plasmonics 2016, 12, 843-848. [CrossRef]

14. Jang, L.-W.; Ju, J.-W.; Jeon, J.-W.; Jeon, D.-W.; Choi, J.-H.; Lee, S.-J.; Jeon, S.-R.; Baek, J.-H.; Sari, E.; Demir, H.V.; et al. Enhanced optical characteristics of light emitting diodes by surface plasmon of Ag nanostructures. Quantum Sens. Nanophotonic Devices VIII 2011, 31, 794511.

15. Lee, J.W.; Ha, G.; Park, J.; Song, H.G.; Park, J.Y.; Lee, J.; Cho, Y.H.; Lee, J.L.; Kim, J.K.; Kim, J.K. AlGaN Deep-Ultraviolet Light-Emitting Diodes with Localized Surface Plasmon Resonance by a High-Density Array of $40 \mathrm{~nm}$ Al Nanoparticles. ACS Appl. Mater. Interfaces 2020, 12, 36339-36346. [CrossRef]

16. Kojima, K.; Yoshida, Y.; Shiraiwa, M.; Awaji, Y.; Kanno, A.; Yamamoto, N.; Chichibu, S. 1.6-Gbps LED-based ultraviolet communication at $280 \mathrm{~nm}$ in direct sunlight. In Proceedings of the 2018 European Conference on Optical Communication (ECOC), Rome, Italy, 23-27 September 2018; pp. 1-3.

17. Sun, X.; Zhang, Z.; Chaaban, A.; Ng, T.K.; Shen, C.; Chen, R.; Yan, J.; Sun, H.; Li, X.; Wang, J.; et al. 71-Mbit/s ultraviolet-B LED communication link based on 8-QAM-OFDM modulation. Opt. Express 2017, 25, 23267-23274. [CrossRef]

18. Okamoto, K.; Niki, I.; Scherer, A.; Narukawa, Y.; Mukai, T.; Kawakami, Y. Surface plasmon enhanced spontaneous emission rate of InGaN/GaN quantum wells probed by time-resolved photoluminescence spectroscopy. Appl. Phys. Lett. 2005, 87, 071102. [CrossRef]

19. Fattal, D.; Fiorentino, M.; Tan, M.; Houng, D.; Wang, S.Y.; Beausoleil, R.G. Design of an efficient light-emitting diode with 10 GHz modulation bandwidth. Appl. Phys. Lett. 2008, 93, 243501. [CrossRef]

20. Chen, H.; Fu, H.; Lu, Z.; Huang, X.; Zhao, Y. Optical properties of highly polarized InGaN light-emitting diodes modified by plasmonic metallic grating. Opt. Express 2016, 24, A856-A867. [CrossRef]

21. Archambeault, B.; Brench, C.; Ramahi, O.M. The Finite-Difference Time-Domain Method; Springer: Boston, MA, USA, 2001; pp. 35-70.

22. Ryu, H.-Y.; Choi, I.-G.; Choi, H.-S.; Shim, J.-I. Investigation of Light Extraction Efficiency in AlGaN Deep-Ultraviolet LightEmitting Diodes. Appl. Phys. Express 2013, 6, 062101. [CrossRef]

23. Yu, G.; Wang, G.; Ishikawa, H.; Umeno, M.; Soga, T.; Egawa, T.; Watanabe, J.; Jimbo, T. Optical properties of wurtzite structure $\mathrm{GaN}$ on sapphire around fundamental absorption edge $(0.78-4.77 \mathrm{eV})$ by spectroscopic ellipsometry and the optical transmission method. Appl. Phys. Lett. 1997, 70, 3209-3211. [CrossRef]

24. Nami, M.; Feezell, D.F. Optical properties of plasmonic light-emitting diodes based on flip-chip III-nitride core-shell nanowires. Opt. Express 2014, 22, 29445-29455. [CrossRef] [PubMed]

25. Homeyer, E.; Mattila, P.; Oksanen, J.; Sadi, T.; Nykänen, H.; Suihkonen, S.; Symonds, C.; Tulkki, J.; Tuomisto, F.; Sopanen, M.; et al. Enhanced light extraction from InGaN/GaN quantum wells with silver gratings. Appl. Phys. Lett. 2013, 102, 081110. [CrossRef]

26. Shin, C.H.; Shin, E.Y.; Kim, M.H.; Lee, J.H.; Choi, Y. Nanoparticle scattering layer for improving light extraction efficiency of organic light emitting diodes. Opt. Express 2015, 23, A133-A139. [CrossRef] [PubMed] 
27. Kuo, Y.; Su, C.Y.; Hsieh, C.; Chang, W.Y.; Huang, C.A.; Kiang, Y.W.; Yang, C.C. Surface plasmon coupling for suppressing p-GaN absorption and TM-polarized emission in a deep-UV light-emitting diode. Opt. Lett. 2015, 40, 4229-4232. [CrossRef] [PubMed]

28. Nami, M.; Feezell, D. Optical properties of Ag-coated GaN/InGaN axial and core-shell nanowire light-emitting diodes. J. Opt. 2015, 17, 025004. [CrossRef]

29. Wirth, R.; Mayer, B.; Kugler, S.; Streubel, K. Fast LEDs for polymer optical fiber communication at $650 \mathrm{~nm}$. In Optoelectronic Devices: Physics, Fabrication, and Application II; SPIE: Bellingham, WA, USA, 2005.

30. Waltereit, P.; Brandt, O.; Trampert, A.; Grahn, H.T.; Menniger, J.; Ramsteiner, M.; Reiche, M.; Ploog, K.H. Nitride semiconductors free of electrostatic fields for efficient white light-emitting diodes. Nature 2000, 406, 865-868. [CrossRef] [PubMed]

31. Podlipskas, Ž.; Aleksiejūnas, R.; Kadys, A.; Mickevičius, J.; Jurkevičius, J.; Tamulaitis, G.; Shur, M.; Shatalov, M.; Yang, J.; Gaska, R. Dependence of radiative and nonradiative recombination on carrier density and Al content in thick AlGaN epilayers. J. Phys. D Appl. Phys. 2016, 49, 145110. [CrossRef] 